\title{
Tropical Jucys covers
}

\author{
Marvin Anas Hahn ${ }^{1}$. Danilo Lewanski ${ }^{2}$
}

Received: 17 March 2020 / Accepted: 1 December 2021 / Published online: 27 January 2022

(c) The Author(s) 2022

\begin{abstract}
We study monotone and strictly monotone Hurwitz numbers from a bosonic Fock space perspective. This yields to an interpretation in terms of tropical geometry involving local multiplicities given by Gromov-Witten invariants. Furthermore, this enables us to prove that a main result of Cavalieri-Johnson-Markwig-Ranganathan is actually equivalent to the Gromov-Witten/Hurwitz correspondence by Okounkov-Pandharipande for the equivariant Riemann sphere.
\end{abstract}

\section{Contents}

1 Introduction . . . . . . . . . . . . . . . . . . . . . . . . 1720

1.1 Structure of the paper . . . . . . . . . . . . . . . . . . . . . . . . . . 1721

2 Preliminaries . . . . . . . . . . . . . . . . . . . . . . . . 1721

2.1 Semi-infinite wedge formalism . . . . . . . . . . . . . . . . . . . . 1721

2.2 Hurwitz numbers in the semi-infinite wedge formalism . . . . . . . . . . . . . . . 1723

2.3 Tropical curves . . . . . . . . . . . . . . . . . . . . . . . . . . . . . . . . . 1724

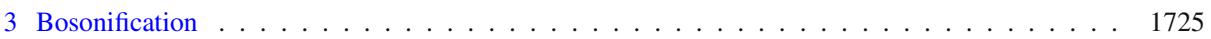

3.1 Newton's identities: $\sigma$ and $h$ polynomials in terms of power sums $p \ldots \ldots$. . . . . . . 1725

3.2 Lascoux-Thibon operator: power sums $p$ of the content in terms of $\mathcal{F}$ operators . . . . . . . 1726

3.3 Boson-Fermion correspondence: $\mathcal{F}$ operators in terms of $\alpha$ operators . . . . . . . . . . . . . 1727

3.4 Putting the pieces together . . . . . . . . . . . . . . . . . . . . . . . . . . . 1729

4 Tropicalisation . . . . . . . . . . . . . . . . . . . . . . . . . . . . . 1729

4.1 Comparison with the tropical curves obtained in $[12,13] \ldots$. . . . . . . . . . . . . . . . . . 1732

5 Gromov-Witten theory and tropical curves . . . . . . . . . . . . . . . . . . . . . 1733

References . . . . . . . . . . . . . . . . . . . . . . . . . 1737

$\bowtie$ Danilo Lewanski

danilo.lewanski@ihes.fr

Marvin Anas Hahn

marvinanashahn@gmail.com

1 Mathematisches Institut, Universität Tübingen, Auf der Morgenstelle 10, 72076 Tübingen, Germany

2 Université de Genève, rue de Conseil-Général 7, 1205 Genève, Switzerland 


\section{Introduction}

Hurwitz numbers and Gromov-Witten invariants with target the Riemann sphere enumerate certain maps between Riemann surfaces. The last two decades have shown several fruitful interactions between those two notions. One of the key revelations for these interactions is the so-called Gromov-Witten/Hurwitz $(G W / H)$ correspondence-found by Okounkov and Pandharipande in [22] — which is a substition rule that relates (completed cycles) Hurwitz numbers and stationary descendant Gromov-Witten invariants. One of the key components of the $\mathrm{GW} / \mathrm{H}$ correspondence is the notion of the Fock space, which gives an operator theoretic interpretation of Gromov-Witten invariants and Hurwitz numbers (for more, see [17,21]).

One successful approach to Hurwitz numbers has been achieved by means of tropical geometry, in which Hurwitz numbers are expressed as enumeration of maps between metric graphs with discrete data (tropical covers) $[5,8,9]$. This interpretation has given rise to many interesting insights, such as a study of polynomial behaviour of double Hurwitz numbers [9] or the introduction of tropical mirror symmetry for elliptic curves involving quasi-modularity statements of certain tropical covers [4]. Furthermore, it was observed in [10] that tropical geometry gives rise to a graphical interface for the Gromov-Witten theory of curves. In particular, it was proved for the case of stationary descendant Gromov-Witten invariants with target $\mathbb{P}^{1}$ that the Fock space notion in [22] is strongly related to the tropical expression derived in [10]. The tropical covers are weighted by local multiplicities, which are GromovWitten invariants themselves.

In recent years many variants of Hurwitz numbers have started to appear in the literature and have grown in importance, also in relation with Chekhov-Eynard-Orantin topological recursion theory. Among those we focus on two, namely monotone and strictly monotone Hurwitz numbers. Monotone Hurwitz numbers appear in the context of random matrix theory as coefficients of large $N$ expansion of the Harish-Chandra-Itzykson-Zuber model, whereas strictly monotone Hurwitz numbers enumerate certain types of Grothendieck dessins d'enfant. These numbers have been intensively studied in terms of operators acting on the Fock space. Furthermore, special cases of these enumerations may be expressed by means of tropical geometry as well $[12,13]$. These expressions however differ fundamentally from the tropical covers involved in [10]. It is natural to ask whether there exists a tropical interpretation involving local Gromov-Witten multiplicities as in the classical case [10].

The aim of this paper is threefold. Starting from previous work on monotone and strictly monotone Hurwitz numbers in terms of the fermionic Fock space $[3,6,14,18]$, we derive an expression of these variants of Hurwitz numbers in terms of the bosonic Fock space.

We then use the connection between bosonic operators and tropical geometry studied in $[7,10]$ to answer the previously posed question positively. In fact we derive a new tropical expression for monotone and strictly monotone Hurwitz numbers that shares several features with the results of [10], as the tropical covers are once again weighted by local multiplicities given by Gromov-Witten invariants. This new interpretation has the advantage that the curves involved carry less non-geometric information than the ones in [12,13] and are closer to the tropical curves involved in $[4,9]$. This new interpretation allows applications in sequel work related to wall-crossing behaviour [15] and mirror symmetry [16].

We use the operator formalism to express a generating series enumerating tropical covers as the vacuum expectation value of an operator. The operator depends on a variable $u$ that keeps track of the Euler characteristics of the tropical covers. A closer inspection of the Euler characteristics leads to degree concentration result: the vacuum expectation value is a 
monomial in $u$. This, in turn, establishes an equivalence between Theorem 5.3.4 in [10] and the original GW/H correspondence of [22].

\subsection{Structure of the paper}

In Sect. 2, we introduce the basic notions revolving around the relation between Hurwitz numbers, Gromov-Witten theory, the Fock space and tropical geometry needed for our work. In Sect. 3 we derive a bosonic expression for monotone and strictly monotone Hurwitz numbers from the fermionic one. Our main result lies in Sect. 4, where we express monotone and strictly monotone Hurwitz numbers in terms of tropical covers with local multiplicities given by Gromov-Witten invariants. Lastly, in Sect. 5 we analyse and compare the original [22] and the tropical [10] version of Gromov-Witten/Hurwitz correpondence via the semiinfinite wedge formalism.

\section{Preliminaries}

In this section, we recall the basic notions regarding the Fock space and tropical geometry, needed for our work. A concise introduction to the needed Gromov-Witten theory can be found in [10, section 2].

\subsection{Semi-infinite wedge formalism}

We introduce the operators needed for the derivation of our results. For a self-contained introduction to the infinite wedge space formalism, we refer the reader to [17,22], where most relevant objects are defined.

Let us define $\varsigma(z)=2 \sinh (z / 2)=e^{z / 2}-e^{-z / 2}$ and $\mathcal{S}(z)=\varsigma(z) / z$. We are particularly interested in the expansion of

$$
\frac{1}{\varsigma(z)}=\frac{1}{2 \sinh (z / 2)}=\sum_{l=0} c_{2 l-1} z^{2 l-1}=\frac{1}{z}-\frac{1}{24} z+\frac{7}{5760} z^{3}+O\left(z^{5}\right)
$$

The coefficients $c_{l}$ have several well known combinatorial and geometric interpretations. The first coefficient is $c_{-1}=1$, and

$$
\begin{aligned}
c_{2 l-1} & =-\frac{\left(2^{2 l-1}-1\right)}{2^{2 l-1}} \frac{B_{2 l}}{(2 l) !}=(-1)^{l} \int_{\overline{\mathcal{M}}_{l, 1}} \lambda_{l} \psi_{1}^{2 l-2} \\
& =\left\langle\tau_{2 l-2}(\omega)\right\rangle_{l, 1}^{\mathbb{P}^{1}}, \quad \text { for } l>0,
\end{aligned}
$$

where:

(i) $B_{k}$ is the $k$-th Bernoulli number, defined by the generating series $\frac{t}{e^{t}-1}=\sum_{k=0}^{\infty} B_{k} \frac{t^{k}}{k !}$.

(ii) $\overline{\mathcal{M}}_{l, 1}$ is the moduli space of stable curves of genus $l$ and one marked point $p$. The class $\lambda_{l}:=c_{l}(\mathbb{E})$ is the top Chern class of the Hodge bundle $\mathbb{E} \rightarrow \overline{\mathcal{M}}_{l, 1}$, which is the rank $l$ vector bundle with fiber $H^{0}\left(C, \omega_{C}\right)$ over $[C, p]$. The class $\psi_{1}=c_{1}(\mathbb{L})$ is the first Chern class of the cotangent line bundle $\mathbb{L} \rightarrow \overline{\mathcal{M}}_{l, 1}$ that has fiber $T_{C, p}^{*}$ over the moduli point $[C, p]$. 
(iii) Recall that for two partitions $\nu, \mu$ of same size $d=|\mu|=|\nu|$, the relative GromovWitten invariant with target $\mathbb{P}^{1}$ is defined as

$$
\left\langle v, \tau_{k_{1}}(\omega) \tau_{k_{2}}(\omega) \cdots \tau_{k_{n}}(\omega), \mu\right\rangle_{g, n}^{\mathbb{P}^{1}}:=\int_{\left[\overline{\mathcal{M}}_{g, n}\left(\mathbb{P}^{1}, v, \mu, d\right)\right]^{v i r}} \prod_{i=1}^{n} e v_{i}^{*}(\omega) \psi_{i}^{k_{i}},
$$

where $\overline{\mathcal{M}}_{g, n}\left(\mathbb{P}^{1}, v, \mu, d\right)$ is the moduli space of stable maps to $\mathbb{P}^{1}$ relative to the partitions $v$ and $\mu,\left[\overline{\mathcal{M}}_{g, n}\left(\mathbb{P}^{1}, v, \mu, d\right)\right]^{v i r}$ is its virtual fundamental class, $\psi_{i}$ is again $c_{1}$ of the cotangent line bundle over the $i$-th marked point, the map $e v_{i}$ : $\overline{\mathcal{M}}_{g, n}\left(\mathbb{P}^{1}, v, \mu, d\right) \rightarrow \mathbb{P}^{1}$ is the $i$-th evaluation morphism that sends the moduli point $\left[C, p_{1}, \ldots, p_{n}, f\right]$ to $f\left(p_{i}\right)$, with $f: C \rightarrow \mathbb{P}^{1}$ the stable relative map, and, finally, $\omega$ denotes the class of a point in $\mathbb{P}^{1}$. For a complete introduction on the topic we recommend [24]. When the partitions are omitted and the degree is not specified, degree zero and empty partitions are meant. The superscripts "०" or "॰" refer to the connected or the not necessarily connected (also called disconnected for simplicity) Gromov-Witten invariant, and correspond in the definition to connected or not necessarily connected (disconnected) stable maps in the moduli space, respectively.

Let $V=\bigoplus_{i \in \mathbb{Z}+1 / 2} \mathbb{C} \underline{i}$ be an infinite-dimensional complex vector space with a basis labeled by half-integers, written as $\underline{i}$. The semi-infinite wedge space or Bosonic Fock space $\mathcal{V}:=$ $\bigwedge^{\frac{\infty}{2}} V$ is defined as the $\mathbb{C}$-vector space spanned by vectors

$$
\underline{k_{1}} \wedge \underline{k_{2}} \wedge \underline{k_{3}} \wedge \cdots
$$

such that for large $i, k_{i}+i-\frac{1}{2}$ equals a constant, called the charge, imposing that $\wedge$ is antisymmetric. The charge-zero sector

$$
\mathcal{V}_{0}=\bigoplus_{n \in \mathbb{N}} \bigoplus_{\lambda \vdash n} \mathbb{C} v_{\lambda}
$$

is then the span of all of the semi-infinite wedge products $v_{\lambda}=\lambda_{1}-\frac{1}{2} \wedge \lambda_{2}-\frac{3}{2} \wedge \cdots$ for integer partitions $\lambda$. The space $\mathcal{V}_{0}$ has a natural inner product $(\cdot, \bar{\cdot} \cdot)$ defined by declaring its basis elements to be orthonormal. The element corresponding to the empty partition $v_{\emptyset}$ is called the vacuum vector and denoted by $|0\rangle$. Similarly, we call the covacuum vector its dual in $\mathcal{V}_{0}^{*}$, and denote it by $\langle 0|$. If $\mathcal{P}$ is an operator acting on $\mathcal{V}_{0}$, we denote with $\langle\mathcal{P}\rangle^{\bullet}$ the evaluation $\langle 0|\mathcal{P}| 0\rangle$.

For $k$ a half-integer, define the operator $\psi_{k}$ by $\psi_{k}:\left(\underline{i_{1}} \wedge \underline{i_{2}} \wedge \cdots\right) \mapsto\left(\underline{k} \wedge \underline{i_{1}} \wedge \underline{i_{2}} \wedge \cdots\right)$, and let $\psi_{k}^{\dagger}$ be its adjoint operator with respect to $(\cdot, \cdot)$. The normally ordered products of $\psi$-operators

$$
E_{i, j}:= \begin{cases}\psi_{i} \psi_{j}^{\dagger}, & \text { if } j>0 \\ -\psi_{j}^{\dagger} \psi_{i} & \text { if } j<0\end{cases}
$$

are well-defined operators on $\mathcal{V}_{0}$. For $n$ any integer, and $z$ a formal variable, define the operators

$$
\begin{aligned}
\mathcal{E}_{n}(z) & =\sum_{k \in \mathbb{Z}+\frac{1}{2}} e^{z\left(k-\frac{n}{2}\right)} E_{k-n, k}+\frac{\delta_{n, 0}}{\varsigma(z)}, \quad \tilde{\mathcal{E}}_{0}(z)=\sum_{k \in \mathbb{Z}+\frac{1}{2}} e^{z k} E_{k, k}, \\
\alpha_{n} & =\mathcal{E}_{n}(0)=\sum_{k \in \mathbb{Z}+\frac{1}{2}} E_{k-n, k} .
\end{aligned}
$$


Their commutation formulae are known to be

$$
\left[\mathcal{E}_{a}(z), \mathcal{E}_{b}(w)\right]=\varsigma(a w-b z) \mathcal{E}_{a+b}(z+w) \text { for } z \neq 0 \text { or } w \neq 0,
$$

and otherwise

$$
\left[\alpha_{k}, \alpha_{l}\right]=k \delta_{k+l, 0} .
$$

The commutation relations involving $\tilde{\mathcal{E}}_{0}$ are clearly the same as the ones involving $\mathcal{E}_{0}$. The coefficients of both operators will play a crucial role in the rest of the paper:

$$
\mathcal{F}_{l}:=\left[z^{l}\right] \tilde{\mathcal{E}}_{0}(z)=\sum_{k \in \mathbb{Z}+1 / 2} \frac{k^{l}}{l !} E_{k, k}, \quad \mathcal{F}_{l}^{s h}:=\left[z^{l}\right] \mathcal{E}_{0}(z)=\sum_{k \in \mathbb{Z}+1 / 2} \frac{k^{l}}{l !} E_{k, k}+c_{l} .
$$

The operator $C=\mathcal{F}_{0}$ is called the charge operator, as its eigenvalues on basis vectors are given by the charge. In particular, it acts as zero on $\mathcal{V}_{0}$. The operator $E=\mathcal{F}_{1}$ is called the energy operator. Observe that the commutation relation for $\alpha$ operators implies that

$$
\left\langle\prod_{j=1}^{\ell(\mu)} \alpha_{\mu_{j}} \prod_{i=1}^{\ell(\nu)} \alpha_{-v_{i}}\right\rangle^{\bullet}=|\operatorname{Aut}(\mu)| \prod_{i=1}^{m} \mu_{i} \cdot \delta_{\mu, v}
$$

where $|\operatorname{Aut}(\mu)|=\prod_{i=1}^{\infty} m_{i}(\mu)$ !, and $m_{i}(\mu)$ the number of parts equal to $i$ in the partition $\mu$.

\subsection{Hurwitz numbers in the semi-infinite wedge formalism}

We are now ready to express the Hurwitz numbers in terms of the semi-infinite wedge formalism. The monotone and strictly monotone Hurwitz numbers have respectively the following expressions, derived in [3]. We use these expressions as definitions. For $g \in \mathbb{Z}_{\geq 0}$, two partitions $\mu$ and $v, m=2 g-2+\ell(\mu)+\ell(\nu)$, let

$$
\begin{aligned}
& h_{g ; \mu, v}^{\leq, \bullet}:=\frac{\left[u^{m}\right]}{\prod \mu_{i} \prod v_{j}}\left\langle\prod_{i=1}^{m} \alpha_{\mu_{i}} \mathcal{D}^{(h)}(u) \prod_{j=1}^{n} \alpha_{-v_{j}}\right\rangle^{\bullet}, \\
& h_{g ; \mu, v}^{<, \bullet}:=\frac{\left[u^{m}\right]}{\prod \mu_{i} \prod v_{j}}\left\langle\prod_{i=1}^{m} \alpha_{\mu_{i}} \mathcal{D}^{(\sigma)}(u) \prod_{j=1}^{n} \alpha_{-v_{j}}\right\rangle^{\bullet},
\end{aligned}
$$

where the operators $\mathcal{D}^{(h)}(u), \mathcal{D}^{(\sigma)}(u)$ depending on the formal variable $u$ have the vectors $v_{\lambda}$ as eigenvectors with eigenvalues the generating series of complete homogeneous polynomials $h$ and elementary symmetric polynomials $\sigma$, evaluated in the content $\mathbf{c r}^{\lambda}$ of the Young diagram associated to the partition $\lambda$ :

$$
\mathcal{D}^{(h)}(u) v_{\lambda}=\sum_{v=0} h_{v}\left(\mathbf{c r}^{\lambda}\right) u^{v} v_{\lambda}, \quad \mathcal{D}^{(\sigma)}(u) v_{\lambda}=\sum_{v=0} \sigma_{v}\left(\mathbf{c r}^{\lambda}\right) u^{v} v_{\lambda}
$$

Remember that the content of a box $(i, j)$ in the Young tableau of a partition is given by $\operatorname{cr}_{(i, j)}=j-i$, and the content $\mathbf{c r}^{\lambda}$ of a partition $\lambda$ is the multiset of all contents of boxes in its Young diagram (cr stands for column-row). For example, the partition $(3,2)$ has boxes $(1,1),(1,2),(1,3),(2,1)$, and $(2,2)$, so $\mathbf{c r}^{(3,2)}=\{0,1,2,-1,0\}$. The connected monotone and strictly monotone Hurwitz numbers are defined in the same way by taking the connected vacuum expectation on the right-hand side. The connected expectation is defined from the disconnected one by means of the inclusion-exclusion formula [11, section 2]. 


\subsection{Tropical curves}

We introduce now the concepts of tropical curve and tropical cover, and we describe the relation between Fock space vacuum expectations and their tropical counterparts. This relation is sometimes (see e.g. [10]) indicated in tropical geometry with the motto

\section{"Bosonification is Tropicalisation."}

A detailed introduction to tropical covers can be found in [1].

Definition 1 An abstract tropical curve is a connected metric graph $\Gamma$ with unbounded edges called ends, together with a function associating a genus $g(v)$ to each vertex $v$. Let $V(\Gamma)$ be the set of its vertices. Let $E(\Gamma)$ and $E^{\prime}(\Gamma)$ be the set of its internal (or bounded) edges and its set of all edges, respectively. The set of ends is therefore $E^{\prime}(\Gamma) \backslash E(\Gamma)$, and all ends are considered to have infinite length. The genus of an abstract tropical curve $\Gamma$ is $g(\Gamma):=h^{1}(\Gamma)+\sum_{v \in V(\Gamma)} g(v)$, where $h^{1}(\Gamma)$ is the first Betti number of the underlying graph. An isomorphism between tropical curves is an isormorphism between the underlying graph that respects edges' lengths and vertices' genera. The combinatorial type of a tropical curve is obtained by disregarding its metric structure.

Definition 2 A tropical cover is a surjective harmonic map $\pi: \Gamma_{1} \rightarrow \Gamma_{2}$ between abtract tropical curves as in [1], i.e.:

(i) Let $V\left(\Gamma_{i}\right)$ denote the vertex set of $\Gamma_{i}$, then we require $\pi\left(V\left(\Gamma_{1}\right)\right) \subset V\left(\Gamma_{2}\right)$;

(ii) Let $E^{\prime}\left(\Gamma_{i}\right)$ denote the edge set of $\Gamma_{i}$, then we require $\pi^{-1}\left(E^{\prime}\left(\Gamma_{2}\right)\right) \subset E^{\prime}\left(\Gamma_{1}\right)$;

(iii) For each edge $e \in E^{\prime}\left(\Gamma_{i}\right)$, denote by $l(e)$ its length. We interpret $e \in E^{\prime}\left(\Gamma_{1}\right), \pi(e) \in$ $E^{\prime}\left(\Gamma_{2}\right)$ as intervals $[0, l(e)]$ and $[0, l(\pi(e))]$, then we require $\pi$ restricted to $e$ to be a linear map of slope $\omega(e) \in \mathbb{Z}_{\geq 0}$, that is $\pi:[0, l(e)] \rightarrow[0, l(\pi(e))]$ is given by $\pi(t)=\omega(e) \cdot t$. We call $\omega(e)$ the weight of $e$. If $\pi(e)$ is a vertex, we have $\omega(e)=0$.

(iv) For a vertex $v \in \Gamma_{1}$, let $v^{\prime}=\pi(v)$. We choose an edge $e^{\prime}$ adjacent to $v^{\prime}$. We define the local degree at $v$ as

$$
d_{v}=\sum_{\substack{e \in \Gamma_{1} \\ \pi(e)=e^{\prime}}} \omega_{e} .
$$

We require $d_{v}$ to be independent of the choice of edge $e^{\prime}$ adjacent to $v^{\prime}$. We call this fact the balancing or harmonicity condition.

We furthermore introduce the following notions:

(i) The degree of a tropical cover $\pi$ is the sum over all local degrees of pre-images of any point in $\Gamma_{2}$. Due to the harmonicity condition, this number is independent of the point in $\Gamma_{2}$.

(ii) For any end $e$, we define a partion $\mu_{e}$ as the partition of weights of the ends of $\Gamma_{1}$ mapping to $e$. We call $\mu_{e}$ the ramification profile above $e$.

We give in the following a formulation of Wick's theorem that suits better our purposes. In fact, Wick's theorem is generally expressed in terms of Feynman graphs, which can then be regarded as tropical covers (for more details see, e.g., [7], last section). We state the result in terms of tropical covers, directly.

Proposition 2.1 (Wick's theorem) Let $\mu$ and $v$ be two partitions of the same size. Consider any collection of non-empty finite sets of non-zero integers $\boldsymbol{x}_{1}, \ldots, \boldsymbol{x}_{n}$ s.t. for each $j$ we have 
$\sum_{x_{i, j} \in x_{j}} x_{i, j}=0$. Denote by $x_{i}^{+}\left(x_{i}^{-}\right)$the tuple of positive (resp. negative) entries of $x_{i}$. Then the vacuum expectation

$$
\left\langle\prod_{t=1}^{\ell(\mu)} \alpha_{\mu_{t}} \prod_{0>x_{i, 1} \in \boldsymbol{x}_{1}} \alpha_{x_{i, 1}} \prod_{0<x_{i, 1} \in \boldsymbol{x}_{1}} \alpha_{x_{i, 1}} \ldots \ldots \prod_{0>x_{i, n} \in \boldsymbol{x}_{n}} \alpha_{x_{i, n}} \prod_{0<x_{i, n} \in \boldsymbol{x}_{n}} \alpha_{x_{i, n}} \prod_{j=1}^{\ell(\nu)} \alpha_{-v_{j}}\right\rangle
$$

is equal to

$$
\sum_{\pi \in \Gamma\left(\mathbb{P}_{\text {trop }}^{1} ; \mu, v\right)} \frac{1}{|\operatorname{Aut}(\pi)|} \prod_{i=1}^{n}\left|\operatorname{Aut}\left(x_{i}^{+}\right)\right|\left|\operatorname{Aut}\left(x_{i}^{-}\right)\right| \prod_{e \in E(\Gamma)} \omega_{e}
$$

where $\Gamma\left(\mathbb{P}_{\text {trop }}^{1} ; \mu, v\right)$ is the set of tropical covers $\pi: \Gamma \longrightarrow \mathbb{P}_{\text {trop }}^{1}=\mathbb{R}$ such that

(i) The unbounded left (resp. right) pointing ends of $\Gamma$ have weights given by the partition $\mu$ (resp. v).

(ii) $|V(\Gamma)|=n$. Let $\left\{v_{1}, \ldots, v_{n}\right\}$ be the set of its vertices ordered linearly from left to right. The local structure at $v_{j}$ is determined by $x_{j}$. For each operator $\alpha_{x_{j, i}}$, we draw an edge germ of weight $\left|x_{j, i}\right|$, which points to the left for $x_{j, i}<0$ and to the right $x_{j, i}>0$. In particular, the valence $\operatorname{val}\left(v_{j}\right)$ of $v_{j}$ is equal to $\left|\boldsymbol{x}_{j}\right|=\sum_{l=1}^{\ell\left(\boldsymbol{x}_{j}\right)} \boldsymbol{x}_{j, l}$.

(iii) $|E(\Gamma)|=\sum_{j}\left|x_{j}\right| / 2$. Every element of $E(\Gamma)$ is formed by connecting one edge germ pointing to the right with one edge germ with same weight but pointing to the left. Viceversa, every edge germ that does not correspond to an unbounded edge (i.e. does not correspond to an element of $v$ or $\mu$ ) must be matched to another edge germ of the same weight $\omega_{e}$ to form an internal edge $e \in E(\Gamma)$. This means in particular that each internal operator $\alpha_{x_{j, i}}$ must be matched with an operator $\alpha_{x_{k, l}=-x_{i, j}}$.

(iv) For the genus of each vertex, we have $g\left(v_{i}\right)=0$.

\section{Bosonification}

In this section we derive a bosonic expression for monotone and strictly monotone Hurwitz numbers from the fermionic one. This is done by means of the boson-fermion correspondence. The fermionic expression itself is recovered from the fermionic expression for the power sums via transformations at the level of symmetric functions.

\subsection{Newton's identities: $\sigma$ and $h$ polynomials in terms of power sums $p$}

Let $\mathbf{X}$ be the set of variables $\left\{X_{1}, \ldots, X_{n}\right\}$. We indicate with $\sigma_{m}, h_{m}$ and $p_{m}$ the symmetric elementary polynomials, the complete homogeneous polynomials and the power sums, respectively:

$\sigma_{m}(\mathbf{X})=\sum_{1 \leq i_{1}<\cdots<i_{m} \leq n} X_{i_{1}} \cdots X_{i_{m}} \quad h_{m}(\mathbf{X})=\sum_{1 \leq i_{1} \leq \cdots \leq i_{m} \leq n} X_{i_{1}} \cdots X_{i_{m}} \quad p_{m}(\mathbf{X})=\sum_{i=1}^{n} X_{i}^{m}$

Newton identities describe relations between the power sums $p_{m}$ and bases $\sigma_{m}$ and $h_{m}$ :

$$
\sigma_{m}(\mathbf{X})=\left[z^{m}\right] \cdot \exp \left(-\sum_{i \geq 1} \frac{p_{i}(\mathbf{X})}{i}(-z)^{i}\right)
$$




$$
h_{m}(\mathbf{X})=\left[z^{m}\right] \cdot \exp \left(\sum_{i \geq 1} \frac{p_{i}(\mathbf{X})}{i} z^{i}\right)
$$

Example 1 Let us test Newton identities for $m=2$ and $n=3$. From the right-hand side of (6) we compute

$$
\begin{aligned}
\operatorname{RHS}(6) & =\frac{(-1)^{3}}{1 !} \frac{p_{2}\left(X_{1}, X_{2}, X_{3}\right)}{2}+\frac{(-1)^{2}}{2 !} p_{1}^{2}\left(X_{1}, X_{2}, X_{3}\right) \\
& =\frac{1}{2}\left[-X_{1}^{2}-X_{2}^{2}-X_{3}^{2}+\left(X_{1}+X_{2}+X_{2}\right)^{2}\right] \\
& =X_{1} X_{2}+X_{1} X_{3}+X_{2} X_{3},
\end{aligned}
$$

which is indeed equal to $\sigma_{2}\left(X_{1}, X_{2}, X_{3}\right)$. From the right-hand side of (7) we compute

$$
\begin{aligned}
\operatorname{RHS}(7) & =\frac{1}{1 !} \frac{p_{2}\left(X_{1}, X_{2}, X_{3}\right)}{2}+\frac{1}{2 !} p_{1}^{2}\left(X_{1}, X_{2}, X_{3}\right) \\
& =\frac{1}{2}\left[X_{1}^{2}+X_{2}^{2}+X_{3}^{2}+\left(X_{1}+X_{2}+X_{2}\right)^{2}\right] \\
& =X_{1} X_{2}+X_{1} X_{3}+X_{2} X_{3}+X_{1}^{2}+X_{2}^{2}+X_{3}^{2},
\end{aligned}
$$

which is indeed equal to $h_{2}\left(X_{1}, X_{2}, X_{3}\right)$.

We can write it more explicitly in terms of ordered partitions $\lambda$. We have

$$
\begin{aligned}
h_{m} & =\left[z^{m}\right] \cdot \exp \left(\sum_{i \geq 1} \frac{p_{i}}{i} z^{i}\right)=\sum_{\lambda \vdash m} \frac{1}{\ell(\lambda) !} \prod_{i}^{\ell(\lambda)} \frac{p_{\lambda_{i}}}{\lambda_{i}}, \\
\sigma_{m} & =\left[z^{m}\right] \cdot \exp \left(-\sum_{i \geq 1} \frac{p_{i}}{i}(-z)^{i}\right)=\sum_{\lambda \vdash m} \frac{(-1)^{m+\ell(\lambda)}}{\ell(\lambda) !} \prod_{i}^{\ell(\lambda)} \frac{p_{\lambda_{i}}}{\lambda_{i}} .
\end{aligned}
$$

\subsection{Lascoux-Thibon operator: power sums $p$ of the content in terms of $\mathcal{F}$ operators}

Let $\mathbf{c r}^{\lambda}$ be the content of the Young diagram associated to the partition $\lambda$, let $p_{k}$ be the $k$-th power sum. We moreover adopt the convention $\mathcal{F}_{r}=0$ for $r \leq 0$, as our calculations all take place in the charge zero sector.

Lemma 3.1 For any partition $\lambda$ we have

$$
\frac{p_{l}\left(\mathbf{c r}^{\lambda}\right)}{l !} v_{\lambda}=\sum_{k=0}^{\infty} c_{2 k-1} \mathcal{F}_{l-(2 k-1)} v_{\lambda} .
$$

Proof 1 of Lascoux and Thibon ([19], proposition 3.3) in our notation as

$$
\left[\frac{\tilde{\mathcal{E}}_{0}(z)}{\varsigma(z)}-E\right] v_{\lambda}=\sum_{l=1}^{\infty} \frac{p_{l}\left(\mathbf{c r}^{\lambda}\right)}{l !} z^{l} v_{\lambda} .
$$

where by definition we have $\tilde{\mathcal{E}}_{0}(z):=\sum_{k \in \mathbb{Z}+1 / 2} e^{z k} E_{k k}=\sum_{r=1} \mathcal{F}_{r} z^{r}$, and $\mathcal{F}_{1}=$ $E$. Therefore subtracting $E$ gets rid of the first term of the expansion $\frac{\tilde{\mathcal{E}}_{0}(z)}{\varsigma(z)}-E=$ $\sum_{l=1} z^{l}\left[\sum_{k=0} c_{2 k-1} \mathcal{F}_{l-(2 k-1)}\right]$. 
Example 2 The first terms read

$$
\begin{aligned}
& \frac{p_{1}\left(\mathbf{c r}^{\lambda}\right)}{1 !} v_{\lambda}=\mathcal{F}_{2} v_{\lambda}, \quad \frac{p_{2}\left(\mathbf{c r}^{\lambda}\right)}{2 !} v_{\lambda}=\left[\mathcal{F}_{3}-\frac{1}{24} \mathcal{F}_{1}\right] v_{\lambda}, \quad \frac{p_{3}\left(\mathbf{c r}^{\lambda}\right)}{3 !} v_{\lambda}=\left[\mathcal{F}_{4}-\frac{1}{24} \mathcal{F}_{2}\right] v_{\lambda}, \\
& \frac{p_{4}\left(\mathbf{c r}^{\lambda}\right)}{4 !} v_{\lambda}=\left[\mathcal{F}_{5}-\frac{1}{24} \mathcal{F}_{3}+\frac{7}{5760} \mathcal{F}_{1}\right] v_{\lambda}, \quad \frac{p_{5}\left(\mathbf{c r}^{\lambda}\right)}{5 !} v_{\lambda}=\left[\mathcal{F}_{6}-\frac{1}{24} \mathcal{F}_{4}+\frac{7}{5760} \mathcal{F}_{2}\right] v_{\lambda} .
\end{aligned}
$$

\subsection{Boson-Fermion correspondence: $\mathcal{F}$ operators in terms of $\alpha$ operators}

The goal of this section is to express the operators we use in terms of sums of strings of $\alpha$ operators weighted by coefficients enriched with a geometric meaning (in fact GromovWitten invariants).

First of all, the famous boson-fermion correspondence (see e.g. [20, section 5] and [23, section 5.2]) gives the following expression for $\mathcal{F}_{l}$ in terms of $\alpha$ operators

$$
\begin{aligned}
\mathcal{F}_{l}= & {\left[z^{l}\right] \cdot \frac{1}{\varsigma(z)} \sum_{s>0} \sum_{n, m \geq 0} \frac{1}{m ! n !}\left(\sum_{\substack{k_{1}+\ldots+k_{m}=s \\
k_{i} \geq 1}} \prod_{i=1}^{m} \frac{\varsigma\left(k_{i} z\right)}{k_{i}} \alpha_{k_{i}}\right) } \\
& \left(\sum_{\substack{\ell_{1}+\cdots+\ell_{n}=s \\
\ell_{i}>0}} \prod_{i=1}^{n} \frac{\varsigma\left(\ell_{i} z\right)}{l_{i}} \alpha_{-\ell_{i}}\right) .
\end{aligned}
$$

Since $\mathcal{F}_{l}^{s h}=\mathcal{F}_{l}+c_{l}$, it is enough to add the $s=0$ term in the sum:

$$
\begin{aligned}
\mathcal{F}_{l}^{s h}= & {\left[z^{l}\right] \cdot \frac{1}{\varsigma(z)} \sum_{s \geq 0} \sum_{n, m \geq 0} \frac{1}{m ! n !}\left(\sum_{\substack{k_{1}+\cdots+k_{m}=s \\
k_{i} \geq 1}} \prod_{i=1}^{m} \frac{\varsigma\left(k_{i} z\right)}{k_{i}} \alpha_{k_{i}}\right) } \\
& \left(\sum_{\substack{\ell_{1}+\cdots+\ell_{n}=s \\
\ell_{i}>0}} \prod_{i=1}^{n} \frac{\varsigma\left(\ell_{i} z\right)}{l_{i}} \alpha_{-\ell_{i}}\right) .
\end{aligned}
$$

Definition 3 Let $S \mathbb{Z}^{k+1-2 g}$ (resp. $S \mathbb{Z}_{s h}^{k+1-2 g}$ ) be the infinite subset of $\mathbb{Z}^{k+1-2 g}$ of integer vectors $\mathbf{x}$ satisfying

$$
x_{1} \leq \cdots \leq x_{l}<0<x_{l+1} \leq \cdots \leq x_{k+1-2 g}, \quad \sum_{i=1}^{k+1-2 g} x_{i}=0, \quad l>0 \quad(\text { resp. } l \geq 0) \text {. }
$$

Let $\mathbf{x}^{-}, \mathbf{x}^{+}$denote the partitions formed by the negative elements multiplied by a minus sign of $\mathbf{x}$, and by the positive elements of $\mathbf{x}$, respectively:

$\mathbf{x}_{i}^{-}=-\mathbf{x}_{i}=-x_{i}, \quad i=1, \ldots, l, \quad \mathbf{x}_{i}^{+}=\mathbf{x}_{l+i}=x_{l+i}, \quad i=1, \ldots, k+1-2 g-l$.

We note that $S \mathbb{Z}^{k+1-2 g}$ and $S \mathbb{Z}_{s h}^{k+1-2 g}$ agree unless $k+1-2 g=0$. If $k+1-2 g=0$, we have $S \mathbb{Z}^{0}=\emptyset$ and $S \mathbb{Z}_{s h}^{0}=\{()\}$, i.e. $S \mathbb{Z}_{s h}^{0}$ contains the empty tuple.

Let us recall the following result, expressing 1-point connected Gromov-Witten correlators with target $\mathbb{P}^{1}$ in terms of $S S$ functions. 
Theorem 3.2 ([22], Theorem 2) For any two partitions $\boldsymbol{x}^{+}$and $\boldsymbol{x}^{-}$of the same size

$$
\begin{aligned}
& \left\langle\boldsymbol{x}^{+}, \tau_{2 g-2+\ell\left(\boldsymbol{x}^{+}\right)+\ell\left(\boldsymbol{x}^{-}\right)},\left.\boldsymbol{x}^{-}\right|_{g} ^{\mathbb{P}^{1}, \circ}\right. \\
& =\frac{1}{\left|\operatorname{Aut}\left(\boldsymbol{x}^{+}\right)\right|\left|\operatorname{Aut}\left(\boldsymbol{x}^{-}\right)\right|}\left[z^{2 g}\right] \frac{\prod_{i=1}^{\ell\left(\boldsymbol{x}_{i}^{+}\right)} S S\left(\boldsymbol{x}_{i}^{+} z\right) \prod_{j=1}^{\ell\left(\boldsymbol{x}^{-}\right)} S S\left(\boldsymbol{x}_{i}^{-} z\right)}{S S(z)} .
\end{aligned}
$$

Lemma 3.3 For the operators $\mathcal{F}_{k}$ and $\mathcal{F}_{k}^{s h}$, we have the following identity

$$
\begin{aligned}
\mathcal{F}_{k} & =\sum_{g=0}^{\infty} \sum_{\boldsymbol{x} \in S \mathbb{Z}^{k+1-2 g}}\left\langle\boldsymbol{x}^{+}, \tau_{k-1}(\omega), \boldsymbol{x}^{-}\right\rangle_{g}^{\mathbb{P}^{1}, \circ} \prod_{0>x_{i} \in \boldsymbol{x}} \alpha_{x_{i}} \prod_{0<x_{j} \in \boldsymbol{x}} \alpha_{x_{j}}, \\
\mathcal{F}_{k}^{s h} & =\sum_{g=0}^{\infty} \sum_{\boldsymbol{x} \in S \mathbb{Z}_{s h}^{k+1-2 g}}\left\langle\boldsymbol{x}^{+}, \tau_{k-1}(\omega),\left.\boldsymbol{x}^{-}\right|_{g} ^{\mathbb{P}^{1}, \circ} \prod_{0>x_{i} \in \boldsymbol{x}} \alpha_{x_{i}} \prod_{0<x_{j} \in \boldsymbol{x}} \alpha_{x_{j}} .\right.
\end{aligned}
$$

Proof Expand $\mathcal{F}_{k}$ by Eq. (8). Observe that we obtain an infinite sum of words in $\alpha$ operators (or strings of $\alpha$ operators), weighted by certain coefficients. The longest possible word has length $k+1$, because $\varsigma(z)^{-1}=z^{-1}+O\left(z^{1}\right)$. Since $\varsigma(z)$ is an odd function in $z$, there are no words in the expansion of $\mathcal{F}_{k}$ of length $k$ with non-zero coefficients. By the same argument, in general the length of the $\alpha$ strings decrease two by two. Let the index $g$ count half the defect of the $\alpha$ strings length. We obtain

$$
\mathcal{F}_{k}=\sum_{g=0}^{\infty} \sum_{\mathbf{x} \in S \mathbb{Z}^{k+1-2 g}}\left[\frac{1}{\left|\operatorname{Aut}\left(\mathbf{x}^{+}\right)\right|\left|\operatorname{Aut}\left(\mathbf{x}^{-}\right)\right|} \frac{\left[z^{k}\right]}{\prod_{\mathbf{x}_{i}^{+}} \mathbf{x}_{i} \prod_{\mathbf{x}_{i}^{-}} \mathbf{x}_{i}} \frac{\prod_{\mathbf{x}_{i}^{+}} \varsigma\left(\mathbf{x}_{i} z\right) \prod_{\mathbf{x}_{i}^{-}} \varsigma\left(\mathbf{x}_{i} z\right)}{\varsigma(z)}\right] \prod_{0>x_{i} \in \mathbf{x}} \alpha_{x_{i}} \prod_{0<x_{j} \in \mathbf{x}} \alpha_{x_{j}}
$$

Note that $(m ! n !)^{-1}$ in Eq. (8) gets substituted by $\left|\operatorname{Aut}\left(\mathbf{x}^{+}\right)\right|\left|\operatorname{Aut}\left(\mathbf{x}^{-}\right)\right|$. This is because we are re-summing $m$-tuples and $n$-tuples in terms of partitions (hence elements are ordered) of length $m$ and $n$ respectively. Substituting $S S(z)=\varsigma(z) / z$ we get

$$
\mathcal{F}_{k}=\sum_{g=0}^{\infty} \sum_{\mathbf{x} \in S \mathbb{Z}^{k+1-2 g}} \frac{1}{\left|\operatorname{Aut}\left(\mathbf{x}^{+}\right) \| \operatorname{Aut}\left(\mathbf{x}^{-}\right)\right|}\left[z^{2 g}\right] \frac{\prod_{\mathbf{x}_{i}^{+}} S S\left(\mathbf{x}_{i} z\right) \prod_{\mathbf{x}_{i}^{-}} S S\left(\mathbf{x}_{i} z\right)}{S S(z)} \prod_{0>x_{i} \in \mathbf{x}} \alpha_{x_{i}} \prod_{0<x_{j} \in \mathbf{x}} \alpha_{x_{j}} .
$$

Applying Theorem 3.2 proves the equation for $\mathcal{F}_{k}$. For $\mathcal{F}_{k}^{s h}$, simply note that the $s=0$ term corresponds to $\left[z^{k}\right] \cdot \frac{1}{\zeta(z)}=c_{k}=\left\langle\emptyset\left|\tau_{2 k-2}(\omega)\right| \emptyset\right\rangle_{k}^{\mathbb{P}^{1}, \circ}$ and therefore corresponds to adding the empty $\alpha$ string, which is what the condition $l \geq 0$ takes care of.

Definition 4 Let us define the operators $\mathcal{G}_{l+1}:=(l-1) ! \sum_{k=0}^{\infty} c_{2 k-1} \mathcal{F}_{l-(2 k-1)}$.

Lemma 3.4 For the operators $\mathcal{G}_{l+1}$, we have the expression

$$
\mathcal{G}_{l+1}=(l-1) ! \sum_{\substack{g_{1}, g_{2}=0 \\ \boldsymbol{x} \in S \mathbb{Z}^{l+2-2 g_{1}-2 g_{2}}}}^{\infty}\left\langle\tau_{2 g_{2}-2}(\omega)\right\rangle_{g_{2}}^{\mathbb{P}^{1}, \circ}\left\langle\boldsymbol{x}^{+}, \tau_{2 g_{1}-2+\ell\left(\boldsymbol{x}^{+}\right)+\ell\left(\boldsymbol{x}^{-}\right)}(\omega),\left.\boldsymbol{x}^{-}\right|_{g_{1}} ^{\mathbb{P}^{1}, \circ} \prod_{0>x_{i} \in \boldsymbol{x}} \alpha_{x_{i}} \prod_{0<x_{j} \in \boldsymbol{x}} \alpha_{x_{j}} .\right.
$$

Proof The proof is a straightforward application of Lemma 3.3 and Eq. (1). 


\subsection{Putting the pieces together}

Putting together what we discussed in Sect. 3.1 and in Sect. 3.2 we obtain

$$
\begin{aligned}
h_{m}\left(\mathbf{c r}^{\rho}\right) v_{\rho} & =\sum_{\lambda \vdash m} \frac{1}{\ell(\lambda) !} \prod_{i}^{\ell(\lambda)}\left(\left(\lambda_{i}-1\right) ! \sum_{k_{i}=0}^{\infty} c_{2 k_{i}-1} \mathcal{F}_{\lambda_{i}-\left(2 k_{i}-1\right)}\right) v_{\rho} \\
\sigma_{m}\left(\mathbf{c r}^{\rho}\right) v_{\rho} & =\sum_{\lambda \vdash m} \frac{(-1)^{m+\ell(\lambda)}}{\ell(\lambda) !} \prod_{i}^{\ell(\lambda)}\left(\left(\lambda_{i}-1\right) ! \sum_{k_{i}=0}^{\infty} c_{2 k_{i}-1} \mathcal{F}_{\lambda_{i}-\left(2 k_{i}-1\right)}\right) v_{\rho} .
\end{aligned}
$$

By the expressions of monotone and strictly monotone Hurwitz numbers in Definition (4) and (5) and by Definition 4 we have

$$
\begin{aligned}
h_{g ; \mu, \nu}^{\leq, \bullet} & =\frac{1}{\prod_{i=1}^{m} \mu_{i} \prod_{j=1}^{n} v_{j}} \sum_{\lambda \vdash m} \frac{1}{\ell(\lambda) !}\left\langle\prod_{i=1}^{\ell(\mu)} \alpha_{\mu_{j}} \prod_{i}^{\ell(\lambda)} \mathcal{G}_{\lambda_{i}+1} \prod_{j=1}^{\ell(\nu)} \alpha_{-v_{j}}\right\rangle^{\bullet} \\
h_{g ; \mu, \nu}^{<, \bullet} & =\frac{1}{\prod_{i=1}^{m} \mu_{i} \prod_{j=1}^{n} v_{j}} \sum_{\lambda \vdash m} \frac{(-1)^{m+\ell(\lambda)}}{\ell(\lambda) !}\left\langle\prod_{i=1}^{\ell(\mu)} \alpha_{\mu_{j}} \prod_{i}^{\ell(\lambda)} \mathcal{G}_{\lambda_{i}+1} \prod_{j=1}^{\ell(\nu)} \alpha_{-v_{j}}\right\rangle^{\bullet}
\end{aligned}
$$

\section{Tropicalisation}

The goal of this section is to express connected monotone and strictly monotone Hurwitz numbers in terms of tropical covers weighted by Gromov-Witten invariants. This is achieved by applying Wick's theorem to the expressions obtained from the bosonification. The main result of the paper is the following.

Theorem 4.1 Let $g$ be a non-negative integer, and $\mu, v$ partitions of the same size $d>0$.

$$
\begin{aligned}
h_{g ; \mu, v}^{\leq, \circ} & =\sum_{\pi \in \Gamma^{\circ}\left(\mathbb{P}_{\text {trop }}^{1}, g ; \mu, v\right)} \frac{1}{|\operatorname{Aut}(\pi)|} \frac{1}{\ell(\lambda) !} \prod_{v \in V(\Gamma)} m_{v} \prod_{e \in E(\Gamma)} \omega_{e} \\
h_{g ; \mu, v}^{<, \circ} & =\sum_{\pi \in \Gamma^{\circ}\left(\mathbb{P}_{\text {trop }}^{1}, g ; \mu, v\right)} \frac{1}{|\operatorname{Aut}(\pi)|} \frac{1}{\ell(\lambda) !} \prod_{v \in V(\Gamma)}(-1)^{1+\operatorname{val}(v)} m_{v} \prod_{e \in E(\Gamma)} \omega_{e}
\end{aligned}
$$

where $\Gamma^{\circ}\left(\mathbb{P}_{\text {trop }}^{1}, g ; \mu, v\right)$ is the set of connected tropical covers $\pi: \Gamma \longrightarrow \mathbb{P}_{\text {trop }}^{1}=\mathbb{R}$ with $b=2 g-2+\ell(\mu)+\ell(v)$ points $p_{1}, \ldots, p_{b}$ fixed on the codomain $\mathbb{P}_{\text {trop }}^{1}$, such that

(i) The unbounded left (resp. right) pointing ends of $\Gamma$ have weights given by the partition $\mu$ (resp. v).

(ii) There exists some $l \leq b$, such that $\Gamma$ has l many vertices. Let $V(\Gamma)=\left\{v_{1}, \ldots, v_{l}\right\}$ be the set of its vertices. Then $\pi\left(v_{i}\right)=p_{i}$. Moreover, let $w_{i}=\operatorname{val}\left(v_{i}\right)$ be the corresponding valences.

(iii) There are two integers associated to each vertex $v_{i}$ of $\Gamma,\left(g_{1}^{i}, g_{2}^{i}\right) \in \mathbb{Z}_{>0}^{2}$ for $i=1, \ldots, l$, such that we have $g\left(v_{i}\right)=g_{1}^{i}+g_{2}^{i}$ for the genus at $v_{i}$ and the following condition holds true

$$
h^{1}(\Gamma)+\sum_{i=1}^{l} g\left(v_{i}\right)=g .
$$


(iv) We define a partition $\lambda$ of length $l$ by $\lambda_{i}=\operatorname{val}\left(v_{i}\right)+2 g\left(v_{i}\right)-2$ and impose the Riemann-Hurwitz condition

$$
\sum_{i=1}^{l} \lambda_{i}=2 g-2+\ell(\mu)+\ell(v) .
$$

(v) For each vertex $v_{i}$, let $\boldsymbol{x}^{+, i}$ (resp. $\boldsymbol{x}^{-, i}$ ) be the right-hand (resp. left-hand) side weights. The multiplicity $m_{v_{i}}$ of $v_{i}$ is defined to be

$$
\begin{aligned}
m_{v_{i}}= & \left(\lambda_{i}-1\right) !\left|\operatorname{Aut}\left(\boldsymbol{x}^{+, i}\right)\right|\left|\operatorname{Aut}\left(\boldsymbol{x}^{-, i}\right)\right|\left(\int_{\overline{\mathcal{M}}_{g_{1}^{i}, 1}\left(\mathbb{P}^{1}, \boldsymbol{x}^{+, i}, \boldsymbol{x}^{-, i},\left|\boldsymbol{x}^{+, i}\right|\right)} \psi^{2 g_{1}^{i}-2+w_{i}} e v_{1}^{\star}(p t)\right) \\
& \left(\int_{\overline{\mathcal{M}}_{g_{2}, 1},{ }_{g_{2}^{i}}} \psi^{2 g_{2}^{i}-2}\right)
\end{aligned}
$$

Proof We will work out the details for the monotone case, as the strictly monotone case is completely parallel. By the previous section, we have

$$
h_{g ; \mu, \nu}^{\leq, \circ}=\frac{1}{\prod_{i=1}^{m} \mu_{i} \prod_{j=1}^{n} v_{j}} \sum_{\lambda \vdash m} \frac{1}{\ell(\lambda) !}\left\langle\prod_{i=1}^{\ell(\mu)} \alpha_{\mu_{j}} \prod_{i}^{\ell(\lambda)} \mathcal{G}_{\lambda_{i}+1} \prod_{j=1}^{\ell(\nu)} \alpha_{-v_{j}}\right\rangle^{\circ} .
$$

A generic summand of this expression is given by

$$
\begin{aligned}
& \frac{1}{\prod_{i=1}^{m} \mu_{i} \prod_{j=1}^{n} v_{j}} \frac{\prod\left(\lambda_{i}-1\right) !}{\ell(\lambda) !} \prod_{i=1}^{\ell(\lambda)}\left\langle\left.\tau_{2 g_{2}^{i}-2}(\omega)\right|_{g_{2}^{i}} ^{\mathbb{P}^{1}, \circ}\right. \\
& \quad \times\left\langle\mathbf{x}^{+, i}, \tau_{2 g_{1}^{i}-2+\ell\left(\mathbf{x}^{+, i}\right)+\ell\left(\mathbf{x}^{-, i}\right)}(\omega), \mathbf{x}^{-, i}\right\rangle_{g_{1}^{i}}^{\mathbb{P}^{1}, \circ}\left\langle\prod_{i=1}^{\ell(\mu)} \alpha_{\mu_{i}} \prod_{i=1}^{\ell(\lambda)} \alpha_{x_{1}^{i}} \cdots \alpha_{x_{\lambda_{i}+1-2 g_{1}^{i}-2 g_{2}^{i}}} \prod_{i=1}^{\ell(\nu)} \alpha_{\nu_{i}}\right\rangle^{\circ},
\end{aligned}
$$

where $g_{1}^{i}, g_{2}^{i} \in \mathbb{Z}_{\geq 0}, x^{i} \in S \mathbb{Z}^{\lambda_{i}+1-2 g_{1}-2 g_{2}}$ and $x_{1}^{i} \leq \cdots \leq x_{l_{i}}^{i}<0<x_{l_{i}+1}^{i} \leq$ $\ldots x_{\lambda_{i}+1-2 g_{1}^{i}-2 g_{2}^{i}}^{i}$ for some $l_{i} \in\left[\lambda_{i}+1-2 g_{1}^{i}-2 g_{2}^{i}\right]$ for all $i \in[\ell(\lambda)]$.

Let us now apply Wick's theorem 2.1 to the last vacuum expectation obtaining consider the vacuum expectation

$$
\begin{aligned}
& \left\langle\prod_{i=1}^{\ell(\mu)} \alpha_{\mu_{i}} \prod_{i=1}^{\ell(\lambda)} \alpha_{x_{1}^{i}} \cdots \alpha_{x_{\lambda_{i}+1-2 g_{1}^{i}-2 g_{2}^{i}}} \prod_{i=1}^{\ell(\nu)} \alpha_{\nu_{i}}\right\rangle^{\circ} \\
& \quad=\sum_{\left.\pi \in \Gamma^{\circ}\left(\mathbb{P}_{\text {trop }}^{1}, 0 ; \mu, \nu\right)\right)} \frac{1}{|\operatorname{Aut}(\pi)|} \prod_{i=1}^{\ell(\lambda)}\left|\operatorname{Aut}\left(x_{i}^{+}\right)\right|\left|\operatorname{Aut}\left(x_{i}^{-}\right)\right| \prod_{e \in E(\Gamma)} \omega_{e},
\end{aligned}
$$

These covers already satisfy conditions $(i)$ and $(i i)$. We note that since we are concerned with connected vacuum expectations, we only need to consider connected tropical covers.

As observed in Proposition 2.1 the vertices of all tropical covers involved are of genus 0. To relate these tropical covers to the desired ones, we associate new genera to the vertices. We start with a fixed generic expression with fixed data $g_{1}^{i}, g_{2}^{i}$ for $i=1, \ldots, \ell(\lambda)$ for a fixed $\lambda$, such that $|\lambda|=2 g-2+\ell(\mu)+\ell(\nu)$.

We associate a new tropical cover to each cover involved in Eq. (9) by setting $g\left(v_{i}\right)=$ $g_{1}^{i}+g_{2}^{i}$. This is obviously a bijection and it preserves automorphisms. We now check that 
$h^{1}(\Gamma)+\sum_{i=1}^{l} g\left(v_{i}\right)=g$. Recall that the Euler characteristic for graphs reads $|V|-|E|=$ $1-h^{1}(\Gamma)$, where $|V|$ is the number of vertices and $|E|$ is the number of edges. We observe $|V|=\ell(\mu)+\ell(v)+\ell(\lambda)$. Moreover, by the handshake lemma, we obtain

$$
\begin{aligned}
|E| & =\frac{1}{2} \sum_{v \in V(\Gamma)} \operatorname{val}(v)=\frac{1}{2}\left(\sum_{i=1}^{\ell(\lambda)}\left(\lambda_{i}+2-2 g\left(v_{i}\right)\right)+\ell(\mu)+\ell(\nu)\right) \\
& =\frac{1}{2}\left(|\lambda|+2 \ell(\lambda)-2 \sum g\left(v_{i}\right)+\ell(\mu)+\ell(\nu)\right)
\end{aligned}
$$

and substituting $|\lambda|=2 g-2+\ell(\mu)+\ell(\nu)$, we obtain

$$
\begin{aligned}
|E| & =\frac{1}{2}\left(2 g-2+2 \ell(\mu)+2 \ell(\nu)+2 \ell(\lambda)-2 \sum_{i=1}^{l} g\left(v_{i}\right)\right) \\
& =g-1+\ell(\mu)+\ell(\nu)+\ell(\nu)-\sum_{i=1}^{l} g\left(v_{i}\right) .
\end{aligned}
$$

Thus by imposing the Euler characteristic constraint, we obtain

$$
\begin{aligned}
& 1-h^{1}(\Gamma)=(\ell(\mu)+\ell(\nu)+\ell(\lambda))-(g-1+\ell(\mu)+\ell(\nu) \\
& \left.+\ell(\lambda)-\sum_{i=1}^{l} g\left(v_{i}\right)\right)=1-g+\sum_{i=1}^{l} g\left(v_{i}\right) .
\end{aligned}
$$

Thus, we obtain $g=h^{1}(\gamma)+\sum_{i=1}^{l}\left(g_{1}^{i}+g_{2}^{i}\right)$ as required, and the new associated tropical covers satisfy condition (iii). Condition (iv) is fulfilled by construction.

Now, we incorporate the prefactor of the above generic summand into the tropical cover as global and local multiplicities. Recall that the global prefactor is

$$
\frac{1}{\prod_{i=1}^{m} \mu_{i} \prod_{j=1}^{n} v_{j}} \frac{\prod\left(\lambda_{i}-1\right) !}{\ell(\lambda) !} \prod_{i=1}^{\ell(\lambda)}\left\langle\tau_{2 g_{2}^{i}-2}(\omega)\right\rangle_{g_{2}^{i}}^{\mathbb{P}^{1}, \circ}\left\langle\mathbf{x}^{+, i}, \tau_{2 g_{1}-2+\ell\left(\mathbf{x}^{+, i}\right)+\ell\left(\mathbf{x}^{-, i}\right)}(\omega), \mathbf{x}^{-}\right\rangle_{g_{1}}^{\mathbb{P}^{1}, \circ}
$$

whereas the tropical covers to be weighted by

$$
\sum_{\pi \in \Gamma\left(\mathbb{P}_{\text {trop }}^{1} ; \mu, \nu\right)} \frac{1}{|\operatorname{Aut}(\pi)|} \prod_{i=1}^{n}\left|\operatorname{Aut}\left(x_{i}^{+}\right)\right|\left|\operatorname{Aut}\left(x_{i}^{-}\right)\right| \prod_{e \in E(\Gamma)} \omega_{e} .
$$

We define the multiplicity of the $i-$ th vertex by

$m_{v_{i}}=\left(\lambda_{i}-1\right) ! \mid \operatorname{Aut}\left(x_{i}^{+}\right) \| \operatorname{Aut}\left(x_{i}^{-}\right)\left\langle\left.\tau_{2 g_{2}^{i}-2}(\omega)\right|_{g_{2}^{i}} ^{\mathbb{P}^{1}, \circ}\left\langle\mathbf{x}^{+, i}, \tau_{2 g_{1}-2+\ell\left(\mathbf{x}^{+, i}\right)+\ell\left(\mathbf{x}^{-, i}\right)}(\omega), \mathbf{x}^{-}\right\rangle_{g_{1}}^{\mathbb{P}^{1}, \circ}\right.$

and we weight each cover by

$$
\frac{1}{\prod \mu_{i} \prod v_{j}} \frac{1}{\ell(\lambda) !} \frac{1}{|\operatorname{Aut}(\pi)|} \prod_{e \in E(\Gamma)} \omega_{e} \prod m_{v_{i}}
$$

to obtain the same contribution. We note that the weights of the unbounded edges of each cover contribute a factor of $\prod \mu_{i} \prod v_{j}$ in the last product, which yields the weight of each cover to be

$$
\frac{1}{\ell(\lambda) !} \frac{1}{|\operatorname{Aut}(\pi)|} \prod \omega_{e} \prod m_{v_{i}}
$$


where the product is taken over all inner edges of $\Gamma$. Finally, we observe that by the same argument as above, any vaccum expectation involved in computing monotone Hurwitz numbers arises from a tropical cover, which concludes the proof of the theorem.

\subsection{Comparison with the tropical curves obtained in $[12,13]$}

In this section, we compare the tropical curves obtained in Theorem 4.1 to the ones derived in $[12,13]$ by means of a particular example. Both these papers use the notion of monotone monodromy graphs associated to the data $(g, \mu, v)$ (for a formal definition of such graphs we refer to the original papers). For us, it is relevant that such graphs carry extra combinatorial data - including each edge being bi-labelled and being coloured in either solid, dashed, or bold.

Theorem 4.2 [12,13] For $g \geq 0$ and $\mu$, v partitions of the same size, we have

$$
h_{g ; \mu, v}^{\leq, \circ}=\frac{1}{\prod \mu_{i}} \sum_{G} \frac{1}{|\operatorname{Aut}(G)|} \prod \omega_{e},
$$

where we sum over all monodromy graphs associated to $(g, \mu, v)$. In this notation $\omega_{e}$ denotes the weight of an edge e of $G$ and the product is taken over a specific subset $H$ of the edges of $G$.

Example 3 (For the data $(g, \mu, v)=(0,(2,1),(3)))$ The data $(g, \mu, v)=(0,(2,1),(3))$ produces only two monodromy graphs, illustrated at the top of Fig. 1. They do not have any non-trivial automorphism, and their subset $H$ consists in both cases of the dashed edge. The weights of the edges are illustrated as numbers in round brackets. Thus we obtain

$$
h_{0 ;(2,1),(3)}^{\leq, \circ}=\frac{1}{1 \cdot 2} \cdot 1+\frac{1}{1 \cdot 2} \cdot 1=1 .
$$

In the language of Theorem 4.1, we obtain the same result by considering a single tropical cover. Its graph is illustrated at the bottom of Fig. 1 -it does not have any automorphism and corresponds to $\lambda=(1)$, which clearly gives $|\ell(\lambda) !|=1$. The local vertex multiplicity can be computed using the relation $c_{2 l-1}=\left\langle\tau_{2 l-2}(\omega)\right\rangle_{l, 1}^{\mathbb{P}^{1}}$ and Theorem 3.2. This yields a vertex multiplicity of 1 and thus

$$
h_{0 ;(2,1),(3)}^{\leq, \circ}=\frac{1}{1} \cdot \frac{1}{1} \cdot 1=1 .
$$

We now compute a slightly more involved example.

Example 4 We consider the case $(g, \mu, v)=(0,(3,1),(3,1))$. Using GAP [2], we compute the monotone Hurwitz number in terms of factorisations in the symmetric group and obtain

$$
h_{0 ;(3,1),(3,1)}^{\leq, \circ}=4 \text {. }
$$

We now show that our interpretation in Theorem 4.1 gives the same number. The three connected graphs for the data $(g, \mu, v)=(0,(3,1),(3,1))$ are illustrated in Fig. 2.

1. For the graph on the left in Fig. 2, we obtain $|\operatorname{Aut}(\pi)|=1$. Further, we see that $\lambda=(1,1)$ and thus $\ell(\lambda) !=2$. For both vertices, we obtain $m_{v}=1$. The only inner edge is of weight $\omega(e)=4$, which yields a contribution of $\frac{1}{1} \cdot \frac{1}{2} \cdot 1 \cdot 4=2$.

2. For the graph in the middle of Fig. 2, we obtain $|\operatorname{Aut}(\pi)|=1$. Again, we obtain $\lambda=(1,1)$ and $\ell(\lambda)=2$. For both vertices, we obtain $m_{v}=1$ and the only inner edge is of weight $\omega(e)=2$. This yields a contribution of $\frac{1}{1} \cdot \frac{1}{2} \cdot 1 \cdot 2=1$. 


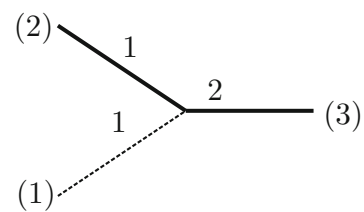

$(2)$
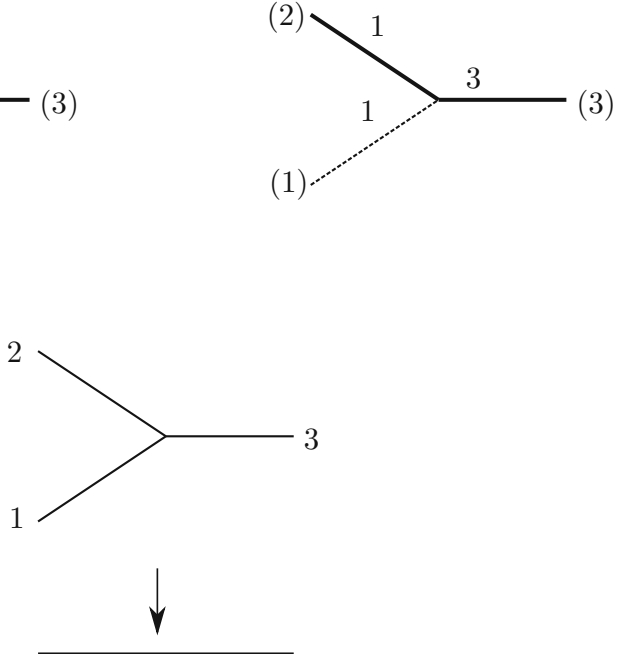

Fig. 1 Comparing the tropical curves appearing in [12,13] (top) to the tropical curves appearing in Theorem 4.1 (bottom) for $(g, \mu, v)=(0,(2,1),(3))$
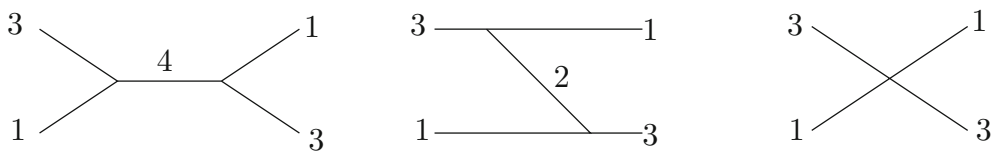

Fig. 2 The tropical curves for $(g, \mu, v)=(0,(3,1),(3,1))$ according to Theorem 4.1

3. For the graph on the right in Fig. 2, we obtain $\mid \operatorname{Aut}(\pi)=1, \lambda=(2)$ and $\ell(\lambda) !=1$. The only vertex gives $m_{v}=1$ and there are no inner edges. Thus, we obtain a contribution of $\frac{1}{1} \cdot \frac{1}{1} \cdot 1 \cdot 1=1$.

In total, we obtain $h_{0 ;(3,1),(3,1)}^{\leq, \circ}=2+1+1=4$, which is what we expected.

\section{Gromov-Witten theory and tropical curves}

The goal of this section is to analyse and compare the original [22] and the tropical [10] version of Gromov-Witten/Hurwitz correpondence via the semi-infinite wedge formalism. The results are summarised at the end of the section.

Definition 5 Let $M_{l}(u)$ be the operator

$$
\begin{aligned}
& \frac{\left[z^{l+1}\right] .}{u^{1 / 2} \varsigma\left(z u^{1 / 2}\right)} \sum_{s \geq 0} \sum_{n, m \geq 0} \frac{1}{m ! n !}\left(\sum_{\substack{\sum_{i=1}^{m} k_{i}=s \\
k_{i} \geq 1}} \prod_{i=1}^{m} \frac{u^{-1 / 2} \varsigma\left(k_{i} z u^{3 / 2}\right)}{k_{i}} \alpha_{k_{i}}\right) \\
& \left(\sum_{\substack{\sum_{i=1}^{n}=s \\
\ell_{i} \geq 1}} \prod_{i=1}^{n} \frac{u^{-1 / 2} \varsigma\left(\ell_{i} z u^{1 / 2}\right)}{l_{i}} \alpha_{-\ell_{i}}\right) .
\end{aligned}
$$


For us it will be relevant that

$$
M_{l}(1)=\mathcal{F}_{l+1}^{s h} .
$$

After expanding it in the same way as in Lemma 3.3, $M_{l}$ takes the form

$$
M_{l}(u)=\sum_{g=0}^{\infty} \sum_{\mathbf{x} \in S \mathbb{Z}_{s h}^{l+2-2 g}}\left\langle\mathbf{x}^{+}, \tau_{l}(\omega), \mathbf{x}^{-}\right\rangle_{g}^{\mathbb{P}^{1}, \circ} u^{\left|\mathbf{x}^{+}\right|+g-1} \prod_{0>x_{i} \in \mathbf{x}} \alpha_{x_{i}} \prod_{0<x_{j} \in \mathbf{x}} \alpha_{x_{j}} .
$$

and therefore coincides with the operator $M_{l}$ defined in [10]. We are now ready to list and compare the two results.

Theorem 5.1 ([10], Theorem 5.3.4)

$$
|\operatorname{Aut}(\mu)||\operatorname{Aut}(v)|\left\langle\mu, \prod_{i=1}^{n} \tau_{k_{i}}(\omega), v\right\rangle^{\mathbb{P}^{1}, \circ}=\frac{\left[u^{g+\ell(\mu)-1}\right]}{\prod_{i} \mu_{i} \prod_{j} v_{j}}\left\langle\prod_{i=1}^{\ell(\mu)} \alpha_{\mu_{j}} \prod_{i}^{n} M_{k_{i}}(u) \prod_{j=1}^{\ell(\nu)} \alpha_{-v_{j}}\right\rangle^{\circ} .
$$

Theorem 5.2 ([22], GW/H correspondence)

$$
|\operatorname{Aut}(\mu)||\operatorname{Aut}(v)|\left\langle\mu, \prod_{i=1}^{n} \tau_{k_{i}}(\omega), v\right\rangle^{\mathbb{P}^{1}, \circ}=\frac{1}{\prod_{i} \mu_{i} \prod_{j} v_{j}}\left\langle\prod_{i=1}^{\ell(\mu)} \alpha_{\mu_{j}} \prod_{i}^{n} M_{k_{i}}(1) \prod_{j=1}^{\ell(\nu)} \alpha_{-v_{j}}\right\rangle^{\circ}
$$

Remark 1 We rearranged GW/H correspondence in a form that is more convenient to our purposes. To derive the formulation above from the original paper [22], simply combine their equation (3.2) with their Proposition 3.1 obtaining

$$
|\operatorname{Aut}(\mu)||\operatorname{Aut}(v)|\left\langle\mu, \prod_{i=1}^{n} \tau_{k_{i}}(\omega), v\right\rangle^{\mathbb{P}^{1}, \bullet}=\frac{1}{\prod_{i} \mu_{i} \prod_{j} v_{j}}\left\langle\prod_{i=1}^{\ell(\mu)} \alpha_{\mu_{j}} \prod_{i}^{n} \mathcal{F}_{k_{i}+1}^{s h} \prod_{j=1}^{\ell(\nu)} \alpha_{-v_{j}}\right\rangle^{\bullet},
$$

then conclude by Eq. (10) and by taking the connected correlators on both sides.

Clearly, in order for both results to hold true, some non-trivial property of the correlator

$$
\left\langle\prod_{i=1}^{\ell(\mu)} \alpha_{\mu_{j}} \prod_{i}^{n} M_{k_{i}}(u) \prod_{j=1}^{\ell(\nu)} \alpha_{-v_{j}}\right\rangle^{\circ}
$$

considered as formal power series $u$ should be involved - in fact, either the degree is completely concentrated in $g+\ell(\mu)-1$, or the sum of all the coefficients, excluding the coefficient of $u^{g+\ell(\mu)-1}$, should vanish altogether. We are going to prove that the first is the correct one.

Proposition 5.3 (Concentration of the degree) Let $K_{0}$ be $\frac{\sum k_{i}+\ell(\mu)-\ell(v)}{2}$.

$$
\left[u^{K}\right]\left\langle\prod_{i=1}^{\ell(\mu)} \alpha_{\mu_{j}} \prod_{i}^{n} M_{k_{i}}(u) \prod_{j=1}^{\ell(\nu)} \alpha_{-v_{j}}\right\rangle^{\circ}=0 \quad \text { for } K \neq K_{0} .
$$

Remark 2 To avoid confusion, let us remark that the statement of the proposition expresses $K_{0}$ as $\left(\sum k_{i}+\ell(\mu)-\ell(v)\right) / 2$ instead of $g+\ell(\mu)-1$ because the variable $g$ loses its meaning without mentioning the GW correlator - however, the two quantities are equal since the genus is determined by the Riemann-Hurwitz condition $\sum k_{i}=2 g-2+\ell(\mu)+\ell(\nu)$.

The proposition immediately implies the following corollary. 
Corollary 5.4 Theorem 5.1 holds true if and only if Theorem 5.2 holds true.

Proof To be precise, what we are going to show is that each vacuum expectation produced by the product of the formal expansions of the $M_{k_{i}}(u)$ in degree different from $K_{0}$ vanishes on its own (i.e. there are no non-trivial cancellations even between summands of the same degree, if that degree differs from $K_{0}$ ). Let us analyse a generic summand of the vacuum expectation $\left\langle\prod_{i=1}^{\ell(\mu)} \alpha_{\mu_{j}} \prod_{i}^{n} M_{k_{i}}(u) \prod_{j=1}^{\ell(v)} \alpha_{-v_{j}}\right\rangle$ expanded via Eq. (11). A generic such summand is of the form

$$
\begin{aligned}
& \prod_{i=1}^{n}\left\langle\mathbf{x}^{+,(i)}, \tau_{k_{i}}(\omega), \mathbf{x}^{-,(i)}\right\rangle_{g_{i}^{\prime}}^{\mathbb{P}^{1}, \circ} \\
& \quad\left\langle\prod_{t=1}^{\ell(\mu)} \alpha_{\mu_{t}} \prod_{i=1}^{n} \alpha_{-\mathbf{x}_{1}^{-,(i)}} \cdots \alpha_{-\mathbf{x}_{p_{i}}^{-,(i)}} \cdot \alpha_{\mathbf{x}_{1}^{+,(i)}} \cdots \alpha_{\mathbf{x}_{q_{i}}^{+,(i)}} \prod_{j=1}^{\ell(\nu)} \alpha_{-v_{j}}\right\rangle u^{\sum_{i=1}^{n}\left(p_{i}+g_{i}^{\prime}-1\right)} .
\end{aligned}
$$

Our goal is to show that every non-vanishing summand satisfies the equation

$$
\sum_{i=1}^{n}\left(p_{i}+g_{i}^{\prime}-1\right)=g+\ell(\mu)-1
$$

We provide two different proofs of the concentration of the degree: the first involves the analysis of each vacuum expectation via the commutation relations of the $\alpha$ operators, the second one analyses the tropical curves associated to each vacuum expectation and compute their Euler characteristic.

Proof (Proof via vacuum expectations analysis) The vanishing of each such summand comes from the following lemma, which immediately follows from (3) and the commutation relations (2). It also follows as immediate corollary from condition (iii) of Wick's Theorem 2.1.

Lemma 5.5 For any collection of finite sets of non-zero integers $\boldsymbol{x}_{1}, \ldots, \boldsymbol{x}_{n}$, the vacuum expectation

$$
\left\langle\prod_{t=1}^{\ell(\mu)} \alpha_{\mu_{t}} \prod_{0>x_{i, 1} \in x_{1}} \alpha_{x_{i, 1}} \prod_{0<x_{i, 1} \in x_{1}} \alpha_{x_{i, 1}} \ldots \ldots \prod_{0>x_{i, n} \in x_{n}} \alpha_{x_{i, n}} \prod_{0<x_{i, n} \in x_{n}} \alpha_{x_{i, n}} \prod_{j=1}^{\ell(\nu)} \alpha_{-v_{j}}\right\rangle=0
$$

whenever the following condition is not satisfied:

$$
\left\{\mu_{i}\right\}_{i=1, \ldots, \ell(\mu)} \cup \boldsymbol{x}_{1}^{+} \cup \cdots \cup \boldsymbol{x}_{n}^{+}=\left\{v_{i}\right\}_{i=1, \ldots, \ell(v)} \cup \boldsymbol{x}_{1}^{-} \cup \cdots \cup \boldsymbol{x}_{n}^{-} .
$$

In other words, the set of positive indices appearing in the expression must be equal to the set of the absolute values of the negative indices.

From Lemma 5.5, we know that, for every non-vanishing summand, the set of positive indices must be equal to the set of the absolute values of the negative indices. In particular, the cardinalities of the two sets must be equal. This gives us the additional requirement

$$
\sum_{i=1}^{n} p_{i}+\ell(v)=\sum_{i=1}^{n} q_{i}+\ell(\mu) \quad \text { or, equivalently, }
$$




$$
\sum_{i=1}^{n} p_{i}-\ell(\mu)=\sum_{i=1}^{n} q_{i}-\ell(\nu)
$$

Now, from the definition of $M_{k_{i}}(u)$ operators we get $p_{i}+q_{i}=k_{i}+2-2 g_{i}^{\prime}$, and therefore

$$
\sum_{i=1}^{n} p_{i}+\sum_{i=1}^{n} q_{i}=\sum_{i=1}^{n} k_{i}+2 n-2 \sum_{i=1}^{n} g_{i}^{\prime} .
$$

Imposing the Riemann-Hurwitz constraint $\sum_{i=1}^{n} k_{i}=2 g-2+\ell(\mu)+\ell(v)$, we get

$$
2\left(g-1-\sum_{i=1}^{n} g_{i}^{\prime}\right)=\sum_{i=1}^{n} p_{i}-\ell(\mu)+\sum_{i=1}^{n} q_{i}-\ell(v)-2 n .
$$

Applying the additional requirement (12) and dividing by two gives the desired equation $g-1-\sum_{i=1}^{n} g_{i}^{\prime}=\sum_{i=1}^{n} p_{i}-\ell(\mu)-n \quad$ or, equivalently, $\quad \sum_{i=1}^{n}\left(p_{i}+g_{i}^{\prime}-1\right)=g+\ell(\mu)-1$.

Proof (Proof via tropical curves' Euler characteristic analysis) Let $\pi: \Gamma \longrightarrow \mathbb{P}_{\text {trop }}^{1}=\mathbb{R}$ be the tropical curve associated to the generic summand as above. Let $V(\Gamma), E(\Gamma), h^{1}(\Gamma)$ the set of vertices, the set of edges and the first Betti number of the tropical cover $\Gamma$. Because we are considering connected covers, the Euler characteristic constraint gives

$$
|V(\Gamma)|-|E(\Gamma)|=1-h^{1}(\Gamma) .
$$

Let us compute each ingredient separately in terms of the indices of the generic correlator.

(i) $|V(\Gamma)|=\ell(\mu)+\ell(v)+n$.

(ii) $|E(\Gamma)|=\sum_{i=1}^{n} p_{i}+\ell(v)$. Indeed the sum of the $p_{i}$ counts the incoming edges, and every edge is either an incoming edge for some vertex, or it is an edge of infinite length on the right and therefore must correspond to some part of $v$.

(iii) $h^{1}(\Gamma)=g-\sum_{i=1}^{n} g_{i}$.

Substuting these quantities in the Euler characteristic contraint we obtain

$$
\ell(\mu)+\ell(v)+n-\left(\sum_{i=1}^{n} p_{i}+\ell(v)\right)=1-\left(g-\sum_{i=1}^{n} g_{i}\right),
$$

which is equivalent to

$$
g+\ell(\mu)-1=\sum_{i=1}^{n}\left(p_{i}+g_{i}-1\right)
$$

This concludes the proof of the proposition.

We can summarise the results of this section in the following two points.

(i) It provides another proof of Theorem 5.1 (by combining Theorem 5.2 and Corollary 5.4).

(ii) It allows a clear comparison between Theorem 5.1 and $\mathrm{GW} / \mathrm{H}$ correspondence 5.2 - on the one hand, it shows that Theorem 5.1 is a refined version of the GW/H correspondence, since it discards many summands that contribute trivially and otherwise would be counted. On the other hand, it shows that the formal $u$-variable in Theorem 5.1 is redundant, which is a non trivial fact, and that getting rid of the $u$-variable recovers nothing but $\mathrm{GW} / \mathrm{H}$ correspondence. 
Acknowledgements We would like to thank Hannah Markwig for useful discussions. The work of D.L. is supported by the Max-Planck-Gesellschaft. M.A.H. gratefully acknowledges partial support by DFG SFBTRR 195 "Symbolic tools in mathematics and their applications", project A 14 "Random matrices and Hurwitz numbers" (INST 248/238-1).

Funding Open access funding provided by University of Geneva

Open Access This article is licensed under a Creative Commons Attribution 4.0 International License, which permits use, sharing, adaptation, distribution and reproduction in any medium or format, as long as you give appropriate credit to the original author(s) and the source, provide a link to the Creative Commons licence, and indicate if changes were made. The images or other third party material in this article are included in the article's Creative Commons licence, unless indicated otherwise in a credit line to the material. If material is not included in the article's Creative Commons licence and your intended use is not permitted by statutory regulation or exceeds the permitted use, you will need to obtain permission directly from the copyright holder. To view a copy of this licence, visit http://creativecommons.org/licenses/by/4.0/.

\section{References}

1. Amini, O., Baker, M., Brugallé, E., Rabinoff, J.: Lifting harmonic morphisms I: metrized complexes and Berkovich skeleta. Res. Math. Sci. 2(1), 7 (2015)

2. Awan, A.A.: The GAP Group. GAP - Groups, Algorithms, and Programming, Version 4.9 .3 (2018)

3. Alexandrov, A., Lewanski, D., Shadrin, S.: Ramifications of Hurwitz theory, KP integrability and quantum curves. J. High Energy Phys. 2016(5), 124 (2016)

4. Boehm, J., Bringmann, K., Buchholz, A., Markwig, H.: Tropical mirror symmetry for elliptic curves. J. Die Reine Angew. Math. (Crelles J.) 2017(732), 211-246 (2017)

5. Bertrand, B., Brugallé, E., Mikhalkin, G.: Tropical open Hurwitz numbers. Rend. Semin. Mate. Univ. Padova 125, 157-171 (2011)

6. Borot, G., Do, N., Lewański, D., Karev, M., Moskovsky, E.: Double hurwitz numbers: polynomiality, topological recursion and intersection theory. arXiv:2002.00900 (2020)

7. Block, F., Göttsche, L.: Fock spaces and refined Severi degrees. Int. Math. Res. Not. 2016(21), 6553-6580 (2015)

8. Cavalieri, R., Johnson, P., Markwig, H.: Tropical Hurwitz numbers. J. Algebr. Combin. 32(2), 241-265 (2010)

9. Cavalieri, R., Johnson, P., Markwig, H.: Wall crossings for double Hurwitz numbers. Adv. Math. 228(4), 1894-1937 (2011)

10. Cavalieri, R., Johnson, P., Markwig, H., Ranganathan, D.: A graphical interface for the gromov-witten theory of curves. Algebr. Geometry Salt Lake City 2015, 139-167 (2018)

11. Dunin-Barkowski, P., Kazarian, M., Orantin, N., Shadrin, S., Spitz, L.: Polynomiality of hurwitz numbers, bouchard-marino conjecture, and a new proof of the ELSV formula. Adv. Math. 279, 67-103 (2015)

12. Do, N., Karev, M.: Monotone orbifold Hurwitz numbers. J. Math. Sci. 226(5), 568-587 (2017)

13. Hahn, M.A.: A monodromy graph approach to the piecewise polynomiality of simple, monotone and grothendieck dessins d'enfants double hurwitz numbers. Graphs Combin. 35(3), 729-766 (2019)

14. Hahn, M.A., Kramer, R., Lewanski, D.: Wall-crossing formulae and strong piecewise polynomiality for mixed Grothendieck dessins d'enfant, monotone, and simple double Hurwitz numbers. Adv. Math. 336, 38-69 (2018)

15. Hahn, M.A.: Wall-crossing and recursion formulae for tropical jucys covers. Trans. Am. Math. Soc. 373, 4685-4711 (2020)

16. Hahn, M.A., Ittersum, J.-W.M., van Leid, F.: Triply mixed coverings of arbitrary base curves: Quasimodularity, quantum curves and a mysterious topological recursions. arXiv:1901.03598 (2019)

17. Johnson, P.: Double Hurwitz numbers via the infinite wedge. Trans. Am. Math. Soc. 367(9), 6415-6440 (2015)

18. Kramer, R., Lewański, D., Popolitov, A., Shadrin, S.: Towards an orbifold generalization of zvonkine's $r$-elsv formula. Trans. Am. Math. Soc. 372, 4447-4469 (2019)

19. Lascoux, A., Thibon, J.-Y.: Vertex operators and the class algebras of symmetric groups. J. Math. Sci. 121(3), 2380-2392 (2004)

20. Miwa, T., Jinbo, M., Jimbo, M.: Solitons: Differential Equations, Symmetries and Infinite Dimensional Algebras, vol. 135. Cambridge University Press, Cambridge (2000) 
21. Okounkov, A., Pandharipande, R.: The equivariant Gromov-Witten theory of $\mathbb{P}^{1}$. Ann. Math. 163, 561605 (2006)

22. Okounkov, A., Pandharipande, R.: Gromov-Witten theory, Hurwitz theory, and completed cycles. Ann. Math. 163, 517-560 (2006)

23. Shadrin, S., Spitz, L., Zvonkine, D.: On double hurwitz numbers with completed cycles. J. Lond. Math. Soc. 86(2), 407-432 (2012)

24. Vakil, R.: The Moduli Space of Curves and Gromov-Witten Theory Enumerative Invariants in Algebraic Geometry and String Theory, pp. 143-198. Springer, Berlin (2008)

Publisher's Note Springer Nature remains neutral with regard to jurisdictional claims in published maps and institutional affiliations. 